\title{
Linx
}

Revue des linguistes de l'université Paris X Nanterre

$80 \mid 2020$

L'héritage de Jean Dubois et Françoise Dubois-Charlier

\section{Françoise Dubois-Charlier et la Grammaire Générative}

Françoise Dubois-Charlier and Generative Grammar

Jacqueline Guéron

\section{(2) OpenEdition}

Journals

Édition électronique

URL : http://journals.openedition.org/linx/6223

ISSN : 2118-9692

Éditeur

Presses universitaires de Paris Nanterre

Référence électronique

Jacqueline Guéron, «Françoise Dubois-Charlier et la Grammaire Générative », Linx [En ligne], 80 | 2020 mis en ligne le 10 juillet 2020, consulté le 05 août 2020. URL : http://journals.openedition.org/linx/ 6223

Ce document a été généré automatiquement le 5 août 2020

Département de Sciences du langage, Université Paris Ouest 


\section{Françoise Dubois-Charlier et la Grammaire Générative}

Françoise Dubois-Charlier and Generative Grammar

Jacqueline Guéron

\section{Introduction}

1 Françoise Dubois-Charlier était une linguiste brillante dont le talent et la curiosité scientifique lui ont permis de comprendre, d'expliquer et de contribuer à divers aspects de la linguistique moderne. Ses publications embrassent la psycholinguistique, la grammaire générative transformationnelle, l'interaction de la syntaxe et de la semantique du discours, et enfin, avec Jean Dubois, le lexique du français.

2 Née en 1941, agrégée d'anglais, docteur d'état en linguistique anglaise, Françoise Dubois-Charlier obtient une maittrise de Conférences à l'Université de Paris III Sorbonne avant de devenir Professeur en 1984. Elle poursuit sa carrière à l'Université de Provence jusqu'en 2009.

3 Elle est l'auteur de nombreux articles et ouvrages à visée didactique ou scientifique, tant en linguistique anglaise que française, dans le domaine de la syntaxe, de la sémantique et de la lexicographie. Elle contribue, entre autres par ses traductions d'ouvrages scientifiques, à l'introduction de la linguistique américaine en France dans les années 1960-1970.

4 Directrice ou co-directrice avec son mari Jean-Dubois de publications et d'ouvrages aux éditions Larousse, elle est également co-auteur avec lui de six ouvrages en linguistique française, ainsi qu'auteure, rédactrice ou contributrice de dix dictionnaires d'anglais ou de français.

5 Dans les dernières années de sa vie, elle se consacre, en collaboration avec Jean Dubois, à l'élaboration de grands dictionnaires électroniques du français. 


\section{Les débuts de Françoise Dubois-Charlier : psycholinguistique et alexie}

6 Les premières publications de F. Dubois-Charlier (D-C) portaient sur la psycholinguistique. Après sa thèse de troisième cycle, Etude neurolinguistique de l'alexie "pure", contribution historique et analyse neurolinguistique d'un groupe de 14 alexiques (1970), D-C a publié des articles, notamment dans Langages (1972 et 1976), qui décrivent le cas de «l'alexie pure »: il s'agit de sujets pouvant parler et écrire normalement et même déchiffrer des lettres mais qui sont incapables de lire ou de se relire. Comme le font remarquer E. Weigl et M. Bierwisch dans l'Introduction à Langage 44 (1976), de tels cas montrent clairement la différence, soulignée dans Chomsky (1965), entre la compétence, les règles de la grammaire internalisées dans le cerveau humain, et la performance, qui peut être atteinte ou déréglée en cas d'accident physique.

\section{Françoise Dubois-Charlier et l'introduction de la Grammaire Générative et Transformationnelle en France}

7 Sans cesser de publier des articles sur l'alexie, Dubois-Charlier a entrepris le projet d'introduire la Grammaire Générative Transformationnnelle en France en visant les étudiants de la langue et de la grammaire anglaise. Cette théorie de la grammaire était développée depuis 1957 (date de Syntactic Structures) par N. Chomsky et ses élèves au Massachusetts Institute of Technology (MIT) à Cambridge, MA, Etats-Unis. Dans Elements de linguistique anglaise: syntaxe (1970) et Elements de linguistique anglaise: la phrase complexe et les nominalisations (1971), D-C explique les principes et les mécanismes de la grammaire générative. Elle se base pour ce faire sur les ouvrages de N. Chomsky (1957 et 1965) et J.J. Katz et P.M. Postal (1964) pour la structure de la phrase simple, de R.A. Jacobs et P. Rosenbaum (1968) pour les complétives, et de R.E. Lees (1968) pour les nominalisations.

8 C'était l'âge d'or du développement de la grammaire générative aux Etats-Unis et de son introduction en France. La théorie est arrivée en même temps que la "révolution » de 1968 d'abord à Nanterre, sous l'inspiration de Jean Dubois, et ensuite au tout nouveau Centre universitaire expérimental de Vincennes (Université de Paris 8). Elle a été enseignée au Département de Linguistique de Paris 8 dès 1968 à travers les cours de Richard Kayne et de Nicolas Ruwet. L'Introduction à la grammaire générative de Ruwet est publié justement en 1968. Dans l'introduction de son livre, Ruwet remercie « M. Jean Dubois, avec qui j'ai pu discuter longuement de plusieurs aspects importants de la grammaire générative » et cite dans sa bibliographie plusieurs publications de Jean Dubois portant sur les grammaires structuralistes et transformationnelles. Dans Eléments de la linguistique anglaise I, D-C (1970) cite comme appartenance universitaire le Centre Universitaire expérimental de Vincennes. ${ }^{1}$

9 La grammaire générative (GG) est une grammaire de la phrase qui se base sur la primauté et l'indépendance de sa composante syntaxique. L'interprétation et la prononciation de la phrase sont par contre des composantes interprétatives, dépendantes. Le premier modèle de la GG, que D-C expose, contenait deux types de 
règles syntaxiques. Les règles de ré-écriture telle que (1) - (3), etc., génèrent la structure profonde (structure-p) de la phrase de haut en bas à partir de la règle (1).

(1) P (phrase) : SN (Syntagme Nominal) + SV (Syntagme verbal)

(2) SV : Aux(iliaire) + Groupe Verbal

(3) GV : V + (SN) + (SP) (Syntagme Prépositionnel) + (SP)

etc.

Une deuxième série de règles, les transformations, définies sur l'alignement des catégories de la structure-p, déplacent, éliminent, inversent, ou ajoutent des catégories pour former la structure de surface (structure-s) de la phrase. Le sens de la phrases est associé à la structure-p, les sons (la prononciation) à la structure-s. Cette grammaire est ébauchée dans (4).

(4) LEXIQUE

|

SYNTAXE

Règles de réécriture

|

structure-p : SENS

|

Transformations

|

structure-s : SONS

11 Dans ce modèle, les transformations ( $\mathrm{T}$ ) étaient nombreuses et puissantes. Par exemple, la T Passive qui transformait la phrase sous-jacente à (5a) en (5b) (i) permutait le sujet et le Complément d'objet, (ii) ajoutait un auxiliaire et des éléments de morphologie verbale pour faire un participe passé, et (iii) insérait l'élément by/par à gauche du sujet postposé. Autre transformation, (6b) est dérivé de (6a) par une montée du sujet d'une phrase enchâssée jusqu'à la position sujet de la phrase matrice (où « $t$ » est la trace du SN déplacé).

(5) a. John hit Bill.

(John a frappé Bill)

(5) b. Bill was hit by John.

(Bill a été frappé par Jean)

(6) a. It seems [John to be sick]

(6) b. John seems [t to be sick]

Les tranformations ne changent pas le sens de la phrase établi en structure-p du point de vue de sa valeur de vérité. Si (5a) «John hit Bill» est vrai, (5b) «Bill was hit by John » est également vrai.

Le modèle chomskyen comporte ainsi une structure profonde avec des propriétés précises :

(i) elle représente les relations grammaticales fondamentales (sujet, complément d'object, etc.)

(ii) c'est le niveau où sont appliquées les « contraintes de sélection » lexicales. Dans le lexique, chaque "prédicat » (Verbe, Nom, Adjectif ou Préposition) est associé à des catégories syntaxiques obligatoires ou facultatives. En structure profonde, des items lexicaux sont insérés et assignés aux catégories appropriées.

(iii) elle constitue l'input des règles transformationnelles.

D-C s'est aussi intéressée aux théorie rivales de la grammaire générative de cette époque, connues collectivement sous le nom de la sémantique générative. Au lieu de 
placer la syntaxe avant la sémantique dans la grammaire de la phrase, ces théories prônent le contraire.

15 D-C (1972) expose les hypothèses des tenants de la sémantique générative. Pour G. Lakoff (1965/71), une théorie basée sur la syntaxe ne permet pas d'aboutir à certaines généralisations qui sont importantes, notamment le fait que deux phrases syntaxiquement distinctes, telles que (7a) et (7b) partagent les mêmes contraintes de sélection. Ces phrases devraient donc avoir la même structure-p. Dans (7a-b), une phrase simple en surface aurait une structure complexe abstraite à deux phrases dans la structure de base.

(7) a. Max cut the salami with a knife.

(Max a coupé le salami avec un couteau.)

(7) b. Max used a knife to cut the salami.

(Max a utilisé un couteau pour couper le salami.)

16 Chomsky (1971) a montré par la suite que la généralisation proposée sur la base de la synonymie de (7a-b) n'est pas généralisable. Par exemple, (8a-b) ne peuvent pas partager une structure syntaxique-sémantique commune.

(8) a. Max used his vacation to write an article.

(8) b. * Max wrote an article with his vacation.

17 McCawley (1966/68) propose, contrairement à Chomsky, que les contraintes de sélection ne concernent pas des traits syntaxiques des items lexicaux tels que [+animé], [+humain] [+abstrait]. Elles seraient plutôt sémantiques et porteraient sur des constituants entiers. Ainsi le contraste entre «mon bras saigne» et «*Le bras de la statue saigne » ne se réduit pas à des traits syntaxiques sur le verbe mais relève, plutôt, du sens de l'item lexical.

McCawley voudrait que le modèle inclue même les présuppositions dans des phrases comme (9).

(9) John called Mary a Republican and then SHE insulted HIM².

(Jean a traité Marie de Républicaine et ensuite ELLE l'a insulté LUI)

19 Selon Lakoff (1965/71), les catégories fondamentales ne sont pas syntaxiques mais logiques : il faudrait unir les verbes et les adjectifs sous la rubrique «Prédicat». Par ailleurs les prépositions et les copules ne seraient que la forme superficielle de verbes sous-jacents. Les circonstants de manière, de lieu, de cause, de fréquence, etc. et les quantificateurs viendraient aussi de verbes appartenant à une proposition " supérieure ».

20 Emmon Bach (1968) considère que les Noms sont aussi des Prédicats logiques. Un SN simple comme « linguiste » devrait être représenté sous la forme : «le x tel que x est linguiste ». Bach voudrait aussi que la phrase inclue des opérateurs en structure sousjacente, tels que l'opérateur Générique (tout $=$ "pour tout $\mathrm{x} »)$, Existentiel (un $=$ « il existe un $\mathrm{N} »$ ), Focus, Question, etc. Ces opérateurs ont une portée et lient des variables dans la phrase. ${ }^{3}$

21 Lakoff (1965/71) décompose des items lexicaux simples en items lexicaux hypothétiques complexes dans la structure sous-jacente de la phrase comme dans (10ab).

(10) a. TUER $=[$ mort + inchoatif + causatif $]$

(10) b. PERSUADER = SN1 fait que SN2 est convaincu : [FAIRE, CONVAINCU]. 
Enfin, les sémanticiens générativistes suggèrent que le niveau de la structure profonde défini par Chomsky n'est ni nécessaire ni utile. Les insertions lexicales seraient dispersées parmi les autres transformations.

D-C (1972) fait remarquer l'absence chez ces auteurs d'une grammaire explicite basée sur leurs propositions. Elle conclut que cette " école » de linguistique est finalement plus critique que positive.

En 1975, D-C présente les hypothèses de Fillmore (1966/69 et 1968). Fillmore conteste la représention des fonctions syntaxiques des constituants en structure profonde. Dans (11a) comme dans (11b), Jean a la même fonction syntaxique: le sujet est défini structurellement comme le SN dominé directement par le nœud P (Phrase). Or, les deux $\mathrm{SN}$ ont des fonctions sémantiques différentes : Jean est sémantiquement Agent dans (11a) mais il a une fonction différente, que Fillmore, dans cet article, appelle Datif, dans (11b).

(11) a. Jean a donné un coup à Paul.

Sujet Objet

(11) b. Jean a reçu un coup de Paul.

Sujet Objet

Pour lui, la structure-p doit contenir uniquement les fonctions sémantiques, les fonctions syntaxiques ne paraissant que dans la structure-s. Par exemple, Fillmore associe les deux phrases de (12) avec une unique structure-p à base sémantique (13). A partir de (13), différentes transformations vont promouvoir un des arguments en position sujet, object, etc. pour produire des phrases de surface comme (12a-b).

(12) a. Cette clé ouvrira la porte.

(12) b. Jean ouvrira la porte avec cette clé

(13)

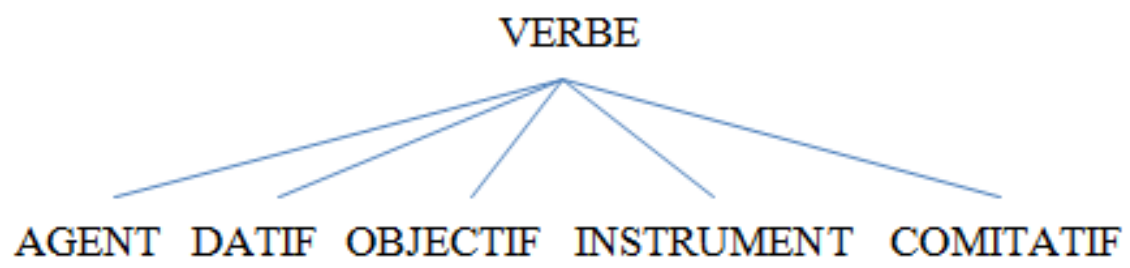

D-C note des problèmes soulevés par cette hypothèse. Elle fait remarquer (avant d'autres chercheurs générativistes) que le sens de la phrase dépend en partie de la structure-s. Par exemple, dans (14) en anglais et (15) en français, la phrase (b), contrairement à la phrase (a), suggère que la totalité de la surface a été traitée.

(14) a. John sprayed paint on the wall.

(14) b. John sprayed the wall with paint.

(15) a. Jean a tapissé de la toile bleue sur le mur.

(15) b. Jean a tapissé le mur de toile bleu.

Ces publications de D-C datent de presque 50 ans. Peuvent-elles avoir un intérêt pour les étudiants de linguistique aujourd'hui ? Certes, de grands changements à la théorie de la GG sont intervenus depuis. En particulier, Chomsky (1993) a introduit le " programme minimaliste». Dans ce cadre, il n'y a plus de règles de ré-écriture: la structure-p disparaît. Les catégories syntaxiques se combinent de façon strictement binaire de bas en haut. Il n'existe plus que deux règles transformationnelles : MERGE 
(« combiner »), qui combine deux catégories pour en faire une seule catégorie plus complexe, et MOVE (« déplacer»), qui déplace des catégories de bas en haut dans la structure. Une Transformation ne peut plus ni effacer, ni ajouter, ni permuter des constituants en cours de dérivation. Des contraintes peu nombreuses, telles que la localité (la cyclicité) contraignent cette opération.

Chomsky (2001) propose que la structure de la phrase se divise en deux phases. La phase inférieure, le syntagme verbal (Sv/vP avec sujet sélectionné ou SV/VP-sans sujet sélectionné) décrit une situation, événement ou état. La phase supérieure (IP-pour la flexion/CP-pour le complémenteur), contient les marqueurs de temps, d'aspect, de modalité, de mode et le complémenteur qui situent l'événement abstrait décrit dans $\mathrm{vP} / \mathrm{VP}$ dans le monde et le temps du discours. Une grammaire minimaliste rend compte de toutes les structures décrites par la première version de la théorie de façon à la fois plus simple et plus explicative.

Malgré ces changements, les principes théoriques soulignés par D-C restent d'actualité. Cette syntacticienne a compris et fait comprendre la supériorité d'une grammaire de type génératif sur les modèles antérieurs: "Les règles de la grammaire de l'anglais contemporain doivent permettre de former et d'expliquer toutes les phrases perçues par les sujets parlant anglais comme appartenant à leur langue» (vol I, p. 11). A partir d'un ensemble d'éléments finis (les catégories), une grammaire générative permet de former l'infinité des phrases grammaticales de la langue et rien que les phrases grammaticales, c'est-à-dire celles acceptées par les locuteurs natifs de la langue. ${ }^{4}$

D-C insiste aussi sur le fait qu'une grammaire générative est explicite. Toutes les phrases de la langue sont associées à une structure syntaxique générée par les règles de la grammaire.

31 Enfin, dans cette grammaire, le lexique et la syntaxe déterminent le sens, ce n'est pas le sens qui détermine la structure. Un Syntagme Nominal n'est pas "le nom d'une personne ou d'une chose ", c'est un constituant avec une structure interne précise et des positions externes précises dans l'arbre syntaxique : un SN doit être contigu à un constituant qui lui assigne un cas morphologique, c'est-à-dire un verbe, une préposition ou la flexion.

32 La première version de la GG plaçait le sens de la phrase uniquement dans la structure profonde, ce qui permettait de rendre compte, par exemple, de la synonymie entre la forme active de la phrase «Jean a bu le vin » et sa forme passive, «Le vin a été bu par Jean ». Il s'ensuit que deux phrases qui ont un sens différent sont associées à une structure-p différente. Chomsky a illustré ce principe avec des paires d'exemples comme (16a), associé à une structure-p comme (17a) vs. (16b), associé à une structure comme (17b).

(16) a. John is eager to please.

(16) b. John is easy to please.

(17) a. John is eager [for himself to please someone].

(17) b. It is easy [ for anyone to please John].

D-C cite une autre paire minimale :

(18) a. I believe John to drive his own car.

(18) b. I convinced John to drive his own car. (vol. II, p. 206)

Des tests syntaxiques montrent si deux phrases doivent être associées à des structures différentes. (17a) et (17b) acceptent tous les deux la $\mathrm{T}$ passive qui déplace un complément d'objet direct à la position de sujet de la phrase. Mais la pronominalisation 
montre que le verbe believe est suivi d'une seul syntagme tandis que le verbe convince est suivi de deux syntagmes.

(19) a. I believe [that].

(19) b. I convinced [John] [of that].

R. Jackendoff (1972 et d'autres études) a montré que seule une partie du sens de la phrase est déterminée en stucture-p, avant les applications des transformations. Une autre partie, déterminée en structure-s, peut même changer la valeur de vérité d'une phrase. Par exemple, la T d'Antéposition de SP génère (20b) à partir de (20a). Dans (20a) Jean veut absolument faire un travail qu'il refuse de faire en (20b).

(20) a. John will do that job for no money.

(Jean fera cette tâche gratuitement)

(20) b. For no money will John do that job

(Aucune somme d'argent ne fera faire cette tâche à Jean)

En fait, c'est la partie de la phrase située dans la phase inférieure de la structure (SV/Sv) qui reste stable. La situation que le syntagme verbal décrit ne change pas en cours de dérivation. Seule peut varier l'insertion (ou l'absence d'insertion) de la situation que le $\mathrm{Sv} / \mathrm{SV}$ décrit dans le monde et le temps du discours, après l'ajout de marqueurs purement grammaticaux de temps, d'aspect, de modalité et de mode. Le choix d'éléments fonctionnels et le déplacement de constituants lexicaux du bas vers le haut de la structure transforment la description d'un type abstrait d'événement en un token d'événement au sein d'une assertion pourvue d'une valeur de vérité.

Les principes de la GG mis en avant par D-C n'ont pas changé depuis 1970-71.

(i) une grammaire générative est récursive

(ii) une grammaire générative est explicite

(iii) le sens d'une phrase dépend du lexique et de la syntaxe

\section{Françoise Dubois-Charlier et les évolutions de la grammaire générative}

Il reste quand même un problème. En voulant introduire la grammaire générative à l'usage des étudiants de la langue anglaise en France, D-C n'a-t-elle pas trop compliqué sa tâche? Car les deux volumes de 1970 et 1971 constituent, en même temps qu'une introduction à la grammaire générative, une grammaire pédagogique de la langue anglaise.

Les travaux de Chomsky et des autres théoriciens de la GG illustraient les principes de la GG par des exemples tirés de l'anglais ou du français, mais sans examiner toutes les constructions de la langue, d'autant moins tous les prédicats verbaux. Le livre French Syntax de R. Kayne, paru en 1975 en anglais et en 1977 en français, citait beaucoup d'exemples du français groupés en des rubriques telles que «la Construction FaireInfinitive » ou «Le placement de clitiques ». Il s'agit dans chaque cas de montrer comment une grammaire générative permet d'établir des relations entre des phrases à l'aide de tranformations simples et de principes généraux tels que l'application « cyclique » des transformations.

Or, comme M. Gross (1979) l'a longuement illustré, certains items lexicaux rejettent certaines opérations syntaxiques ${ }^{5}$. Par exemple, la transformation passive, qui change la structure sous-jacente à une phrase comme (21a) en la structure sous-jacente à la phrase (21b), ne s'applique pas à (22a). 
(21) a. John hit Bill.

(John a frappé Bill.)

(21) b. Bill was hit by John.

(Bill a été frappé par John.)

(22) a. The package weighed ten kilos.

(Le paquet pesait dix kilos.)

(22) b. *Ten pounds were weighed by the package.

(*Dix kilos ont été pesés par le paquet.)

41 S'il est vrai que dans une GG, le lexique et la syntaxe déterminent conjointement la stucture et le sens des phrases, ces composantes sont en partie autonomes. Certains éléments lexicaux, ou plutôt leurs combinaisons, peuvent bloquer des transformations à cause de l'incompatibilité entre leurs traits sémantiques et le sens aspectuo-temporel de la structure-s. Par exemple, appelons « dynamisme » le sens d'un prédicat qui décrit un «changement d'état » de son complément d'objet. L'absence de dynamisme dans la description déterminée par le SV weigh ten kilos/peser dix kilos, comparée au dynamisme impliqué par la relation hit John / frapper John empêche le premier SV d'être associé à l 'output dynamique de la $\mathrm{T}$ passive. Le même contraste entre un SV dynamique et un SV non-dynamique joue un rôle majeur dans d'autres structures contenant un marqueur d'aspect dynamique, telle que la structure progressive dans (23).

(23) a. John is hitting Bill.

(John est en train de frapper Bill).

(23) b. *The package is weighing ten kilos.

( ${ }^{*}$ Le paquet est en train de peser dix kilos)

Les problèmes surviennent moins avec le choix d'un verbe isolé qu'avec le SV que le verbe forme avec son complément, au niveau de l'interprétation de la phase vP/VP. Quand weigh/peser + COD a un sens dynamique comme (24a), la T Passive s'applique parfaitement.

(24) a. John weighed the package carefully.

(John a pesé soigneusement le paquet.)

(24) b. The package was weighed carefully by John.

(Le paquet a été soigneusement pesé par John.)

Apparemment, il faut choisir. Si l'on veut explorer les principes et les mécanismes d'une grammaire générative qui rende compte de la dérivation et de la relation entre un grand nombre de phrases partageant des propriétés lexicales, syntaxiques, et interprétatives, il faut mettre provisoirement de côté l'examen de tous les prédicats de la langue. Ou, si l'on veut examiner toutes les propriétés syntaxiques et sémantiques de tous les verbes ou autres items lexicaux, on peut faire un dictionnaire, en associant, dans la mesure du possible, chaque verbe ou autre prédicat avec l'ensemble des structures syntaxiques et l'ensemble de sens avec lesquels ils peuvent être associés, sans chercher à rendre explicite la grammaire qui produit ces phrases. C'est ce deuxième chemin qu'ont choisi de suivre M. Gross et ensuite J. Dubois et F. Duboischarlier.

Nous parlerons brièvement du Dictionnaire LVF plus loin. 


\section{Françoise Dubois-Charlier et la linguistique de corpus} Cette opération est permise seulement quand le Syntagme prépositionnel (SP) est complément du verbe (ou de V suivi d'une particule), comme dans (26); elle est en général interdite quand le SP est circonstanciel comme dans (27).

(26) a. the brake isn't something 0 you have to think about _-.

(\# le frein n'est pas quelque chose que tu dois penser à __-)

(27) a. The only occasion on which he seemed truly to be a patient.

(La seule occasion à laquelle il semblait être vraiment un patient)

(27) b. * The only occasion which he seemed to be truly a patient on.

Comme aucune structure syntaxique ne figure dans cet essai, l'auteur n'a pas distingué en termes structuraux un complément du verbe «sœur de V » inclus dans le SV noyau d'un circonstanciel contenu dans un SV adjoint au premier SV. 
d'exposer les principes de la grammaire générive. En 1997 elle publie, avec Béatrice d'exposer les principes de la grammaire générative. En 1997 elle publie, avec Béatrice Vautherin, un ouvrage pédagogique sur la syntaxe anglaise en vue des concours de l'enseignement supérieur. Bien que les auteurs mentionnent dans leur avant-propos : "Cette initiation à la syntaxe s'inscrit dans le cadre de la linguistique descriptive anglosaxonne, éminemment représentée par les grammaires de R. Quirk et de son équipe ", on y retrouve les principes de base de la grammaire générative. En 2008 les mêmes auteurs publient un historique de la grammaire générative et transformationnelle. En 2008 encore, dans un volume consacré aux « mots non simples », D-C étudie la manière dont des textes dits «difficiles » dépendent de l'existence et du type de mots dérivés qu'ils contiennent. Elle analyse notamment des transformations de Nominalisation à base de phrases élémentaires, tout à fait dans l'esprit des premiers générativistes, et arrive à distinguer, dans la morpho-syntaxe des nominalisations, les affixes et les opérations transformationnelles faciles des difficiles.

\section{Françoise Dubois-Charlier et les dictionnaires électroniques}

Enfin, D-C est co-auteur avec Jean Dubois de Les Verbes Français (LVF), un dictionnaire électronique qui contient 25610 entrées verbales représentant 12310 verbes différents dont 4188 à plusieurs entrées. Pour chaque verbe sont fournis des schèmes syntaxiques ainsi que les restrictions de sélection sur le sujet et l'objet direct, et les types de complément d'objet, de complément circonstanciel et de préposition. La classification des verbes vise à tenir compte de l'adéquation entre la syntaxe et l'interprétation sémantique, représentée par un schéma syntactico-sémantique sous la rubrique "opérateur».

Quatorze classes génériques sont établies sur une base sémantique, par exemple la Classe $\mathrm{E}:$ « déplacement d'un lieu ou vers un lieu ». Dans chaque classe, les verbes sont divisés en 53 classes sémantico-syntaxiques selon les contraintes de sélection (+/animé), («propre/figuré»), ce qui fait 248 sous-classes sémantiques (ex. E1: «sortir, faire sortir »). S'y ajoutent des sous-types syntaxiques (Ex: E1a : «sortir d'un lieu »+ intransitifs, avec trois variantes de sous-catégorisation).

Comme des commentateurs l'ont déjà noté, ce travail représente un retour à la méthodologie structuraliste de Z. Harris (1952) et de M. Gross $(1968,1975)$. Or, Gross estimait que son travail pour élaborer un dictionnaire électronique est incompatible avec une grammaire générative parce que chaque verbe de la langue a une entrée différente. ${ }^{7}$ M. Gross n'a pas tort. Mais est-ce que la tentative de créer un dictionnaire exhaustif des verbes met en question la construction d'une grammaire de principes et paramètres? Au contraire, ce type de dictionnaire présuppose l'existence d'une grammaire, grammaire structuraliste de Harris pour Gross, grammaire 
transformationnelle de Chomsky pour Dubois et D-C, d'où sont tirées les structures syntaxiques associées à chaque item lexical traité. ${ }^{8}$

Nous n'avons pas examiné ce dictionnaire, dont nous ne doutons pas de l'utilité pour des recherches sur le lexique du français. Nous ferons seulement deux remarques concernant les transformations proposées dans ce travail.

Le LVF associe chaque structure de phrase à des variantes issues de diverses «transformations" qui forment un ensemble de phrases reliées les unes aux autres. Quelques exemples :

(i) L'instrument devient sujet

(28) a. On ennuie Paul avec nos histoires.

(28) b. Nos histoires ennuient Paul.

(ii) Le locatif devient sujet

(29) a. Les moustiques pullulent dans le marais.

(29) b. Le marais pullule de moustiques

61 (iii) L'object direct non-animé devient sujet d'un pronominal passif

(30) a. On enrichit la langue de mots nouveaux.

(30) b. La langue s'enrichit de mots nouveaux.

62 Ces types de transformations à la manière de Harris et de Gross, ne sont pas admis dans le cadre du programme minimaliste, qui reconnaît l'unique transformation « Move $\alpha$ ». Il n'est pas possible de supprimer une Préposition en cours de dérivation pour transformer, par exemple, (28a) ou (29a) en (28b) ou (29b) en supprimant la Préposition avec ou dans; ni de transformer (28a) ou (30a) en (28b) ou (30b) en supprimant le sujet indéfini on. Par ailleurs, dans le modèle actuel de la GG, contrairement au premier modèle, les transformations n'opèrent pas sur une phrase entière. "Move $\alpha$ " s'applique de façon libre à des constituants créés par des applications itérées de l'opération «Merge ». Le sens des phrases, loin d'être associé au seul verbe noyau, se construit graduellement pendant la dérivation, l'interprétation se faisant à la fin de chaque phase $\mathrm{VP} / \mathrm{VP}$ et IP/CP. Le lien syntaxique et sémantique le plus fort se crée entre un verbe et son complément direct, qui peut être une catégorie simple tel qu'un SN ou un SP, ou bien une catégorie plus complexe telle qu'une phrase (IP/CP) finie ou nonfinie. Comme le sens de la phrase dépend de l'accumulation des sens de syntagmes de plus en plus complexes, il faut tenir compte de tous les items lexicaux et de toutes les sous-structures de la phrase pour calculer son interprétation. Il peut arriver que les traits sémantiques d'un verbe ou d'un SV soient sémantiquement incompatibles avec le sens associé à la structure syntaxique finale. Ainsi, la transformation illustrée dans (28) et dans (31) où « l'instrument devient sujet » est refusée dans (32).

(31) a. John lifted the load with a tractor.

(31) b. The tractor lifted the load.

(32) a. John lifted the meat with a fork

(32) b. *The fork lifted the meat.

Dans (29) et dans (33) «un élément locatif devient sujet » mais c'est impossible dans (34).

(33) a. Flies are buzzing in the garden.

(33) $b$. The garden is buzzing with flies

(34) a. Mary is dancing in the garden.

(34) b. *The garden is dancing with Mary. 
Toutes les ressemblances lexico-sémantiques entre des paires de phrase ne relèvent pas de transformations. Il y a beaucoup de façons de dire plus ou moins la même chose. Or, le modèle de la grammaire générative fournit un moyen syntaxique de rapprocher les structures (28)-(30): elles ont la même structure de VP et elles sont sujettes à la même transformation, une application locale de "Move $\alpha$ ». Toutes ces phrases peuvent être associées avec le SV dans (35) de type inaccusatif, qui, contrairement au $\mathrm{SV}$, manque de sujet sélectionné. Il s'avère qu'en anglais comme en français, toute phrase doit avoir un sujet visible en structure-s. Pour satisfaire à cette condition purement syntaxique, un SV inaccusatif donne lieu très généralement à la Montée (par la règle " Move $\alpha$ ») en position sujet d'un des compléments du verbe. Son application au deuxième complément de (28a) et (29a) et au complément d'objet direct de (30a) engendre directement les phrases (29b-30b).

(35) $\left[_{\mathrm{SV}}\right.$ Verbe SN - SN - (SP)]

(35) a. ennuyer Paul (avec) nos histoires

(35) b. pulluler le marais de moustiques

(35) c. enrichir la langue de mots nouveaux

Comme pour les cas de «P-stranding " analysés dans D-C (1999), un SN ou un SP dans un constituant adjoint au SV de base ne peut pas subir la Montée à la position sujet.

(36) a. $\left[{ }_{\mathrm{SV}}[\mathrm{sV}\right.$ ennuie Paul] tous les jours] ]

(36) b. *Tous les jours ennuient Paul.

\section{Conclusion.}

Françoise Charlier-Dubois était un syntacticienne de premier ordre, formée par une étude constante et approfondie de la grammaire générative. Au lieu de se limiter à explorer plus en avant le modèle de la grammaire générative tel qu'il a évolué dans le temps, consciente sans doute de la responsabilité d'un professeur dans un Département de Langues et inspirée par l'important projet de dictionnaire électronique de Jean Dubois, D-C a choisi de mettre ses compétences de syntacticienne au service des études des textes littéraires et du lexique des verbes français. Dans les domaines de la grammaire anglaise, de l'analyse de textes littéraires, et du développement d'un dictionnaire électronique exhaustif des verbes français, ses connaissances syntaxiques et son grand talent de chercheur étaient indispensables.

\section{BIBLIOGRAPHIE}

Bach, E. (1968). "Nouns and Noun Phrases", in E. Bach, R.T. Harms (éds), Universals in Linguistic Theory, pp. 90-122. N.Y.: Holt, Rinehart and Winston.

Balibar-Mrabti, A. (2007). «Phrases simples et exemplification dans Les Verbes Français. Une réflexion sur on comme sujet ", in Langue Française, n 153, pp. 111-126.

Chomsky, N. (1957). Syntactic Structures. La Haye, Mouton (trad. fran. Le Seuil, 1969). 
Chomsky, N. (1962). "A Transformational Approach to Syntax", in A. Hill (éd), Proceedings of the third Texas Conference on Problems of Linguistic Analysis in English, pp. 124-168. Austin: University of Texas Press. [Reprinted in J. Fodor, J. Katz (éds), The structure of language, pp. 211-45. Englewood Cliffs, NJ: Prentice-Hall.]

Chomsky, N. (1965). Aspects of the Theory of Syntax. Cambridge, MA: MIT Press.

Chomsky, N. (1971). "Deep Structure, Surface Structure, and Semantic Interpretation” in D. Steinberg and L. Jakobuvits (éds), Semantics: an Interdisciplinary Reader in Philosophy, Linguistics, and Psychology, pp. 183-216. Cambridge: Cambridge University Press.

Chomsky, N. (1995). The Minimalist Program. Cambridge, MA: MIT Press.

Chomsky, N. (2001). « Derivation by Phase », in M. Kenstowicz (éd), Ken Hale: A Life in Language, pp 1-52. Cambridge, MA: MIT Press.

Dubois-Charlier, F. (1970). Eléments de linguistique anglaise I : syntaxe. Paris : Larousse.

Dubois, J., Dubois-Charlier, F. (1970). « Principes et méthode de l'analyse distributionnelle », in Langages, $\mathrm{n}^{\circ} 20$, pp. 3-13.

Dubois-Charlier, F. (1971). Eléments de linguistique anglaise II : la phrase complexe et les nominalisations. Paris : Larousse.

Dubois-Charlier, F. (1972a). « A propos de l'alexie pure », in Langages, n² 25, pp. 76-94.

Dubois-Charlier, F. (1972b). « La sémantique générative: une nouvelle théorie linguistique ? », in Langages, $\mathrm{n}^{\circ} 27$, pp. 5-77.

Dubois-Charlier, F. (1975). « Avant-propos : Les premiers articles de Fillmore », in Langages, $\mathrm{n}^{\circ} 38$, pp. 3-17.

Dubois, J., Dubois-Charlier, F. (1997). « Synonymie syntaxique et classification des verbes français ", in Langages, $\mathrm{n}^{\circ}$ 128, pp. 51-71.

Dubois-Charlier, F., Vautherin, B. (1997). Syntaxe anglaise. Paris : Vuibert.

Dubois-Charlier, F. (1999). «Les relatives en américain contemporain », in ANGLOPHONIA /

Sigma, $\mathrm{n}^{\circ}$ 6, pp. 61-112.

Dubois-Charlier, F. (2008a). « Du mot non simple au texte non simple » in B. Kalz (éd), Regards croisés sur les mots non simples, pp. 151-163. Lyon : ENS Editions, Collections Langages.

Dubois-Charlier, F., Vautherin, B. (2008b). « La grammaire générative et transformationelle: bref historique ", in La Clé des Langues. Lyon : ENS LYON/DGESCO.

Fillmore, Ch. (1966/69). “Toward a Modern Theory of Case”, in D. A. Reibel and S. A. Schane (éds), Modern Studies in English, pp. 361-375. Englewood Cliffs: Prentice-Hall.

Fillmore, Ch. (1968). “The Case for Case”, in E. Bach, R. Harms (éds), Universals in Linguistic Theory, pp. 1-89. N.Y.: Holt, Reinhart and Winston.

François, J., Le Pesant, D., Leeman, D. (2007). « Présentation de la classification des Verbes Français de Jean Dubois et Françoise Dubois-Charlier », in Langue Française, n 153, pp. 3-19.

Gross, M. (1968). Grammaire transformationnelle du français : syntaxe du verbe. Paris : Larousse.

Gross, M. (1975). Méthodes en syntaxe. Paris : Hermann.

Gross, M. (1979). « On the Failure of Generative Grammar », in Language, n 55, pp. 859-885.

Harris, Z. (1952). « Discourse Analysis », in Language, $\mathrm{n}^{\circ} 28$, pp. 1-30. 
Jackendoff, R. (1972). Semantic Interpretation in Generative Grammar. Cambridge, MA.: MIT Press.

Jacobs, R.A., Rosenbaum, P. (1968). English Transformational Grammar. Waltham, MA.: Blaisdell.

Katz, J.J., Postal, P.M. (1964). An integrated theory of linguistic descriptions. Cambridge, MA.: MIT

Press.

Kayne, R. (1975). French Syntax. Cambridge, MA.: MIT Press. (trad.franc. 1977)

Lakoff, G. (1965/71). Irregularity in Syntax. N.Y.: Holt, Reinhart and Winston.

Lees, R.E. (1960). The Grammar of English Nominalizations. International Journal of English Linguistics, vol. 26, $\mathrm{n}^{\circ} 3$, pt.2. Bloomington, Indiana.

McCawley, J. (1966/68). “Concerning the base component of a transformational grammar”, in Foundations of Language, $\mathrm{n}^{\circ}$ 04, pp. 243-269.

Ruwet, N. (1968). Introduction à la grammaire générative. Paris : Plon.

\section{NOTES}

1. Je suis moi-même entrée à l'Université Paris 8 en 1968 comme chargée de cours en linguistique au Département d'Anglais. J'y ai appris la grammaire générative en suivant les cours de R. Kayne qui venait de soutenir son doctorat en linguistique à MIT sous la direction de N. Chomsky. La même année, Antoinette Balibar-Mrabti, que je remercie d'avoir partagé avec moi ses souvenirs de cette époque, soutenait à Nanterre une Maîtrise sur «La transformation négative dans le français parlé » dirigée par Jean Dubois.

2. Les majuscules indiquent les accents contrastifs.

3. Ces opérateurs existent dans le modèle de la GG. Certains, comme l'opérateur interrogatif, sont groupés dans la partie supérieure de la phrase dans la projection du Complémenteur ( $\mathrm{CP}$ ). La Négation est située dans une projection localisée entre celle du Temps (la Flexion) et le SV. D'autres opérateurs sont associés avec des items lexicaux, comme l'opérateur existentiel inclus dans le déterminant un/a, an, le verbe exister/exist ou l'expression il y a/there is, there are.

4. La recherche des principes de la grammaire d'une langue et de toutes les langues (grammaire universelle) est compatible avec des variations dans la grammaire des individus, car les principes de la grammaire s'accompagnent de paramètres permettant la variation qui sont également des objets d'étude.

5. Gross semble en profond désaccord avec la position de Chomsky (1962:244-45), dont il cite les propos suivants: "There are in fact exceptions to many of the transformational rules given above, perhaps to all. [...] But discovery of exceptions to grammatical generalizations is of no consequence in itself, except when it leads to an alternative more comprehensible generalization. "

6. Dans son abstract D-C dit vouloir " revive a methodology [...] to gather and analyse all the occurrences in a complete discourse [...] as opposed to an approach [...] where the argumentation is theory-based and theory-oriented, and examples collected here and there are mere illustrations of what is posited." La grammaire générative fait appel pour les jugements de grammaticalité surtout aux intuitions des locuteurs natifs. Car le locuteur natif " possède » une grammaire internalisée qui inclut toutes les phrases de la langue, qu'il les ait entendues auparavant ou pas, et pas seulement celles réunies dans un corpus, si étendu soit-il. Ceci dit, un corpus est très utile, même pour le locuteur natif, en ce qu'il peut attirer l'attention de celui-ci vers des structures qu'il n'a pas en tête ou qu'il n'a pas l'habitude d'utiliser, mais qu'il reconnaît comme grammaticales. 
7. Gross (1979, p. 860) note que dans la matrice des 12000 verbes classés par Gross (1975), « no two lexical items have identical syntactic properties » (« Il n'y a pas deux verbes qui partagent les mêmes propriétés syntaxiques »- notre traduction).

8. Pour un exemple de la tension entre un dictionnaire exhaustif et une grammaire générative, voir la discussion dans Balibar-Mrabti (2007) à propos du traitement dans LVF du pronom on.

\section{RÉSUMÉS}

Cet article constitue un bref aperçu du parcours de Françoise Dubois-Charlier, resituant ses travaux dans leur contexte historique, depuis ses débuts en psycholinguistique jusqu'aux dictionnaires électroniques (Les verbes français), en passant bien sûr et avant tout par la grammaire générative et transformationnelle, la sémantique générative, les structuralisme, mais aussi la linguistique de corpus.

This paper provides an overview of Françoise Dubois-Charlier's career, placing her works in their historical context, from her early career in psycholinguistics to her latest works on electronic dictionaries (Les verbes français in this paper), including of course and above all generative and transformational grammar, generative semantics, structuralism, but also corpus linguistics.

\section{INDEX}

Mots-clés : Françoise Dubois-Charlier, grammaire générative et transformationnelle, sémantique générative, linguistique de corpus, structuralisme, Les verbes français Keywords : Françoise Dubois-Charlier, generative and transformational grammar, generative semantics, corpus linguistics, structuralism, Les verbes français

\section{AUTEUR}

\section{JACQUELINE GUÉRON}

Paris 3 Sorbonne Nouvelle, PRISMES 